\title{
ROUGHNESS EFFECTS IN SURFACE ENHANCED RAMAN SCATTERING - EVIDENCE FOR ELECTROMAGNETIC AND CHARGE TRANSFER ENHANCEMENT MECHANISM*
}

\author{
S. KRUSZEWSKI AND J. SKONIECZNY \\ Institute of Mathematics and Physics, Academy of Technology and Agriculture \\ S. Kaliskiego 7, 85-790 Bydgoszcz, Poland \\ (Received April 26, 1991; in final version September 9, 1991)

\begin{abstract}
Investigations of Surface Enhanced Raman Scattering (SERS) of pyridine adsorbed on the silver electrode of different roughness have been performed. The obtained results indicate that the micro- and the atomic scale roughness influence the intensity of SERS signal. This proves for the electromagnetic and charge transfer origin of the enhancement in the SERS phenomenon.
\end{abstract}

PACS numbers: 78.30.-j, 81.60.Bn, 82.65.Pa

\section{Introduction}

The Surface Enhanced Raman Scattering (SERS) phenomenon was discovered in 1974 by Fleischman et al. [1]. The authors investigated the Raman scattering of laser light by pyridine molecules adsorbed on the surface of silver electrode activated by the oxidation-reduction procedure. The obtained results were rather spectacular. The intensity of Raman scattered light appeared to be about $10^{6}$ times larger than one could expect from the number of excited molecules. Thus, one can assume the existence of surface mechanisms, which amplify the Raman scattered light intensity. The study of these mechanisms was the subject of great number of both experimental and theoretical works [2-6]. Numerous theoretical models have been proposed, however, no one of them could explain that effect individually and completely [7]. It was experimentally confirmed that the surface roughness plays

*This work was partially supported by Central Research Program CPBP 01.08 and partially by Ministry of National Education grant P/04/393/90-2. 
a crucial role in the SERS phenomenon. The strong SERS signal is produced only by molecules adsorbed on rough surfaces. At present most of investigators agree that SERS is a result of two mutual complementary roughness-based mechanisms, which can be explained by electromagnetic (EM) and charge transfer (CT) models [6]. The EM model assumes that the experimentally observed enhancement of Raman scattering is a result of the electric field enhancement of both incident and scattered light on the rough surface. The initially smooth surface, submitted to roughness procedure, acquires bumps, which can be approximated to spheres, hemispheres and prolate spheroids. From the solution of electrodynamic equations it follows that the electric field of the incident and scattered light is enhanced inside the micro-objects $[3,8]$. The magnitude of this enhancement depends on the size and shape of micro-objects at the surface and on the imaginary part of the dielectric function of metal surface. The giant gain is theoretically expected when: 1) micro-structures are of small sizes, i.e. the semi-axis of micro-objects are smaller than $10 \mathrm{~nm} ; 2$ ) micro-objects at the surface are prolongated, i.e. $a / b$ is large, where $a, b$ are the semi-axis perpendicular and parallel to the metal macro-surface, respectively; 3) the imaginary part of the dielectric function of the metal for the frequency of exciting light is small (the largest enhancement is obtained when the frequency of the exciting light is equal to the frequency of the localized surface plasmon). The strong SERS signals are mainly observed on rough surfaces and colloidal particles of silver, gold and copper. Silver shows a small value of the imaginary part of the dielectric function in the whole range of visible region, whereas gold and copper in the red region only [9]. To observe the SERS phenomenon on the silver surface one can use the exciting light in the whole visible region. For gold and copper it is necessary to use red light in order to obtain detectable signal. These experimental facts seem to confirm partially the EM model. The observed signal enhancement, however, is larger than that predicted by EM model. One may then expect that another mechanism exists, which is responsible for the additional enhancement. This can be explained by charge transfer (CT) called the "adatom" or the "active sites" model $[2-6,10,11]$ : In this model the existence of adatoms at the surface (i.e. atomic scale roughness) plays an important role. Adatoms have dimensions much smaller than micro-structures needed for EM model. They are represented by single metal atoms or clusters, which can form chemical bonds with the adsorbed molecules. The CT model assumes that: 1 . The incident light causes a transfer of an electron from the metal to the adsorbed molecule; 2 . The electron comes back to the metal, the photon is emitted and the molecule remains in the vibrationally excited state. The probability of these electron transitions is extremely large when the molecules are adsorbed at sites of the atomic scale roughness (i.e. when the adatoms exist at the surface) [3]. It is now generally accepted that both the EM and CT mechanisms determine the experimentally observed signal and that the signal produced by CT mechanism comes from the top of EM structure.

In this work we present the results of investigation of the influence of the electrode potential and the state of surface roughness on SERS signal from pyridine adsorbed on silver electrode. We interpret the obtained results on the basis of EM and CT models. 


\section{Experimental}

SERS investigations were performed in three-electrode electrochemical cell. A scheme of our experimental set-up is presented in Fig. 1. A polished polycrystalline silver disc of $6 \mathrm{~mm}$ diameter was used as a working electrode and placed in the centre of the cylindrical pyrex-glass cell. The circular Pt-wire, placed opposite to the working electrode, was used as a counter electrode. All potentials were referred to the saturated calomel electrode (SCE) located in the proximity of working electrode. The exposed surface of the working electrode was polished to the mirror finish with emery paper and alumina powder and rinsed in acetone and distilled water. The $0.1 \mathrm{M}$ aqueous solution of $\mathrm{KCl}$ containing $0.05 \mathrm{M}$ pyridine was used as electrolyte. The latter one was deoxigenated by bubbling argon into the cell. Prior to SERS measurements the silver electrode was roughened by a few oxidation-reduction cycles (ORCs). The potential during the ORC was changed from +0.15 to $-1.20 \mathrm{~V}$. The SERS spectra were excited with ILA $120-1 \mathrm{Ar}^{+}$ion laser ( $514.5 \mathrm{~nm}$ line). The power of the laser beam was equal to $200 \mathrm{~mW}$. Laser light after passing the filter removing the plasma light was focused on the surface of working electrode at the incident angle equal to $60^{\circ}$. The light scattered on the electrode was focused on the entrance slit of a double grating monochromator. The effective band pass of the monochromator slits was $4 \mathrm{~cm}^{-1}$. The cooled photomultiplier (M12 FQ51) and the photon counting system was used for SERS spectra detection.

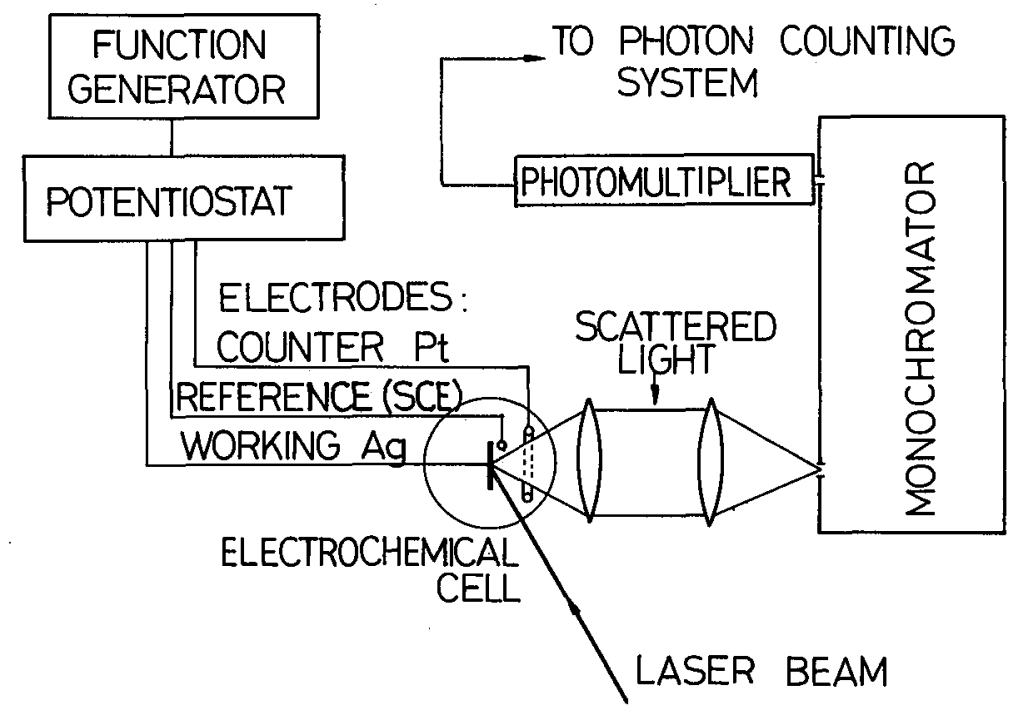

Fig. 1. Scheme of the experimental apparatus. 


\section{Results and discussion}

Figure 2 presents a typical voltammogram recorded during the ORC at a sweep rate of $5 \mathrm{mVs}^{-1}$. Figure 3 shows the SERS spectrum of pyridine adsorbed on $\mathrm{Ag}$ electrode the potential of which was equal to $-0.6 \mathrm{~V}$. This spectrum was

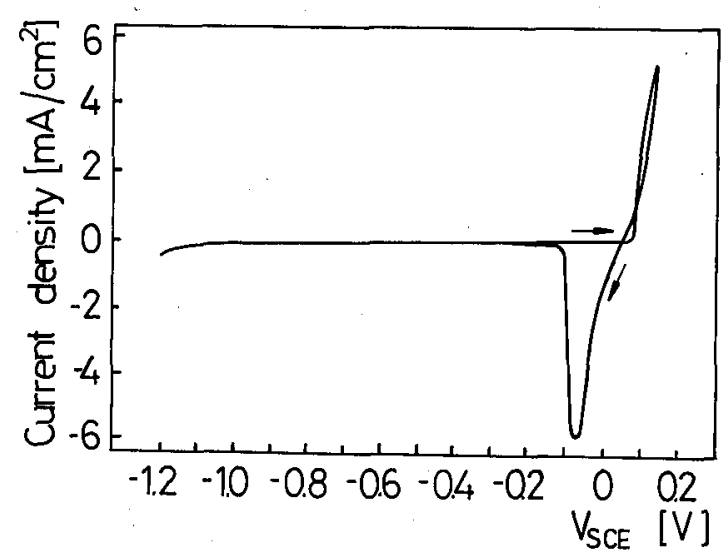

Fig. 2. Cyclic voltammogram of $\mathrm{Ag}$ electrode at scan rate $5 \mathrm{mVs}^{-1}$ in $0.05 \mathrm{M}$ pyridine and $0.1 \mathrm{M} \mathrm{KCl}$ aqueous solution.

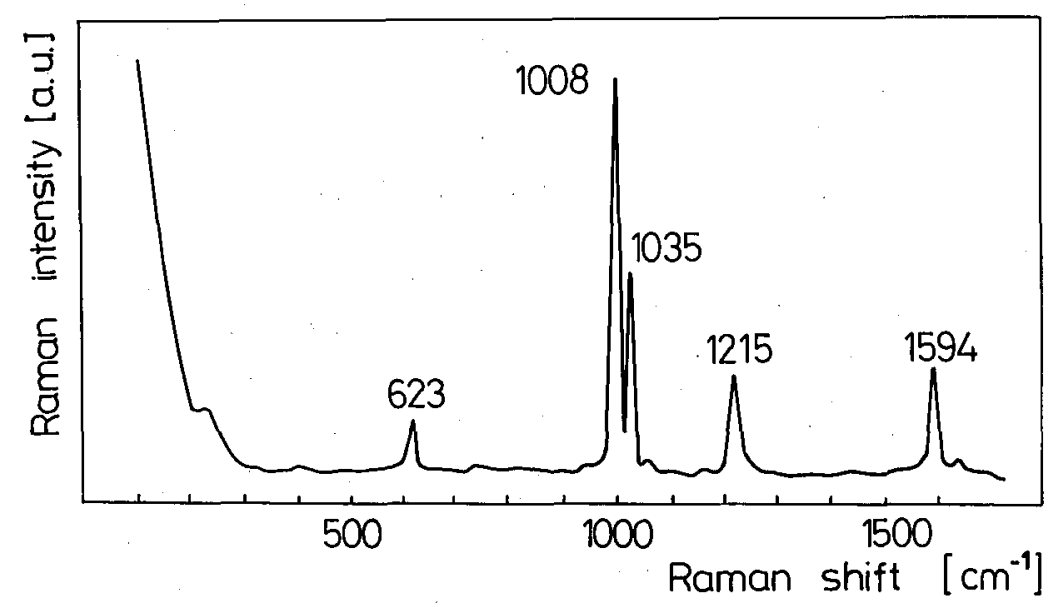

Fig. 3. SERS spectrum of pyridine adsorbed on rough Ag electrode, potential $\mathrm{V}_{\mathrm{SCE}}=$ $-0.6 \mathrm{~V}, 0.1 \mathrm{M} \mathrm{KCl}+0.05 \mathrm{M}$ pyridine aqueous solution, $514.5 \mathrm{~nm}$ excitation line, laser power $200 \mathrm{~mW}$, resolution $4 \mathrm{~cm}^{-1}$.

recorded after performing 5 ORCs at the sweep rate of $5 \mathrm{mVs}^{-1}$. We performed the investigations of the dependence of SERS signal on the electrode potential for all 
separate bands. Results of this measurements as so-called volt-Ramanograms are plotted in Fig. 4. This figure presents also the volt-Ramanogram of the $1025 \mathrm{~cm}^{-1}$

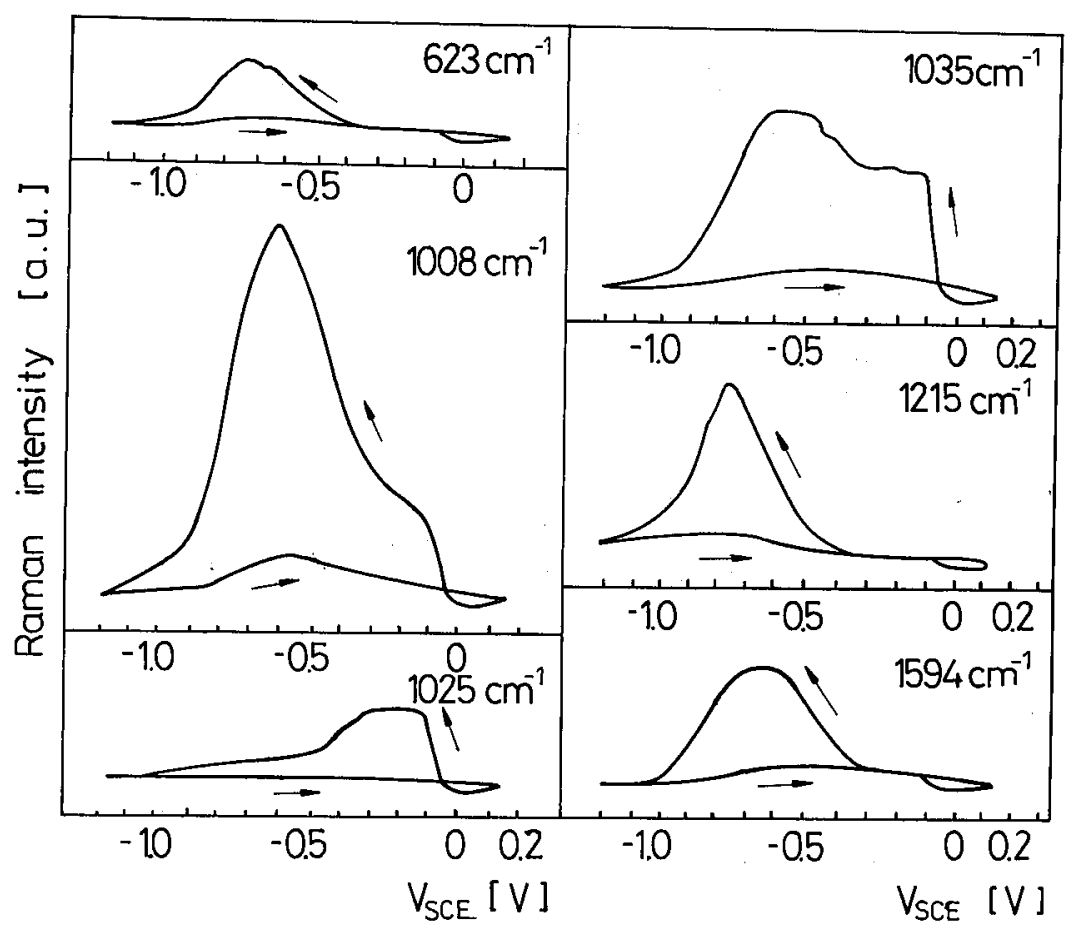

Fig. 4. Cyclic volt-Ramanograms of $\mathrm{Ag}$ electrode in $0.1 \mathrm{M} \mathrm{KCl}+0.05 \mathrm{M}$ pyridine aqueous solution for different bands of SERS spectrum, scan rate $5 \mathrm{mVs}^{-1}$, resolution $4 \mathrm{~cm}^{-1}, 514.5$ nmexcitation laser line.

band which is not shown in Fig. 3, since it appears in the range of the potential from about -0.1 to about $-0.4 \mathrm{~V}$ only. The complete spectrum in the above mentioned range contains three close bands 1008,1025 and $1035 \mathrm{~cm}^{-1}$. As follows from the presented volt-Ramanograms the SERS signal strongly depends on the electrode potential and on the direction of the potential sweep. It is related to the processes occurring on the electrode surface, when the potential is changed. For potentials, at which the current is positive (see Fig. 2), the oxidation of the electrode occurs $\left(\mathrm{Ag} \rightarrow \mathrm{Ag}^{+}+\mathrm{e}^{-}\right)$and $\mathrm{Ag}$ surface becomes partially covered by $\mathrm{AgCl}$ layer. Next, when the current becomes negative, the reduction of $\mathrm{AgCl}$ layer $\left(\mathrm{Ag}^{+}+\mathrm{e}^{-} \rightarrow \mathrm{Ag}^{0}\right)$ begins. The reduced $\mathrm{Ag}^{0}$ atoms called adatoms are surrounded and immobilized by the adsorbed $\mathrm{Cl}^{-}$anions, water and pyridine molecules. During the reduction process the number of adatoms increases and as it is shown in Fig. 4 the SERS signal increases as well. The SERS intensity for the bands 623,1008 , 1035,1215 and $1594 \mathrm{~cm}^{-1}$ is maximal for potentials from -0.6 to $-0.7 \mathrm{~V}$. SERS intensity for the $1025 \mathrm{~cm}^{-1}$ band is maximal in the potential range from -0.2 to $-0.3 \mathrm{~V}$. In accordance with [12] the existence of the $1025 \mathrm{~cm}^{-1}$ band is caused by 
a) Electrolyte

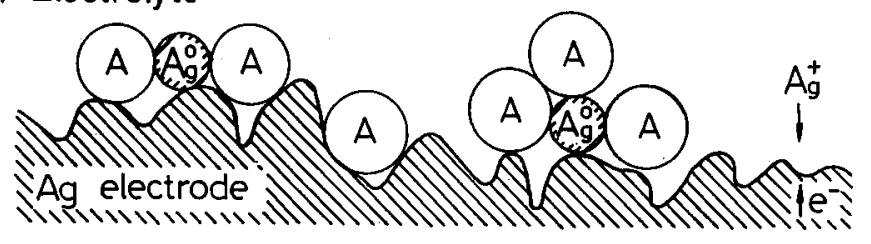

b) Electrolyte
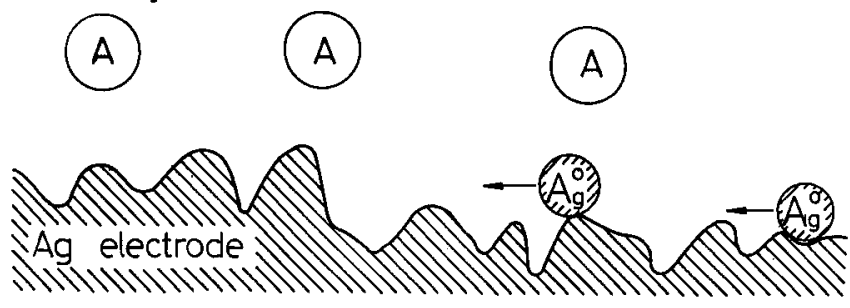

c) Electrolyte

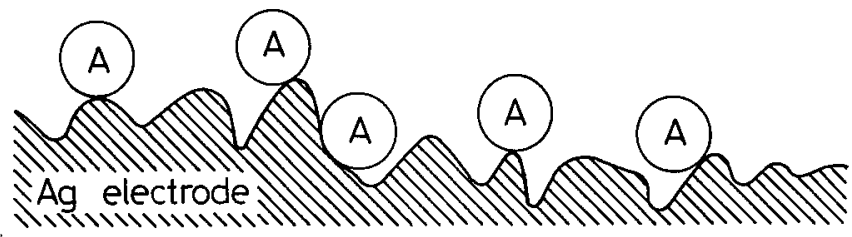

Fig. 5. The physical situation at the $\mathrm{Ag}$ electrode/electrolyte interface at various electrode potential. (A - adsorbate, i.e pyridine molecules, pyridine-water complexes, water molecules and $\mathrm{Cl}^{-}$anions; $\mathrm{Ag}^{0}-\mathrm{Ag}^{0}$ adatoms). (a) $\mathrm{VSCE}_{\mathrm{SC}}=-0.6 \mathrm{~V}$ (decreasing the potential); (b) $\mathrm{V}_{\mathrm{SCE}}=-1.2 \mathrm{~V}$; (c) $\mathrm{V}_{\mathrm{SCE}}=-0.6 \mathrm{~V}$ (increasing the potential).

the molecules of pyridine chemisorbed on silver via nitrogen atom $\left(\mathrm{C}_{5} \mathrm{H}_{5} \mathrm{~N}-\mathrm{Ag}\right)$. Bands 1008, 1035, 623, 1215 and $1594 \mathrm{~cm}^{-1}$ are similar in frequency to those in aqueous solution. From this fact one can suppose that these bands are ascribed to $\mathrm{Py}-\mathrm{H}_{2} \mathrm{O}$ complexes with hydrogen bond $\left(\mathrm{C}_{5} \mathrm{H}_{5} \mathrm{~N}-\mathrm{H} \cdots \mathrm{O}-\mathrm{H}\right)$ rather than to pyridine and that this complex is adsorbed on the surface of silver electrode. The SERS intensity is a function of both the concentration of the adsorbate on the surface and the number of $\mathrm{Ag}^{0}$ adatoms. In the range of potentials from -0.2 to $-0.3 \mathrm{~V}$ the concentration of adatoms as well as pyridine molecules adsorbed via nitrogen atom is large. Changing the potentials toward the more negative ones, pyridine molecules adsorbed via nitrogen atom are desorbed more rapidly than those adsorbed via water. Therefore, the $1025 \mathrm{~cm}^{-1}$ band disappears, although the adatoms concentration is large. In the potential range from -0.6 to $-0.7 \mathrm{~V}$ there is a large 


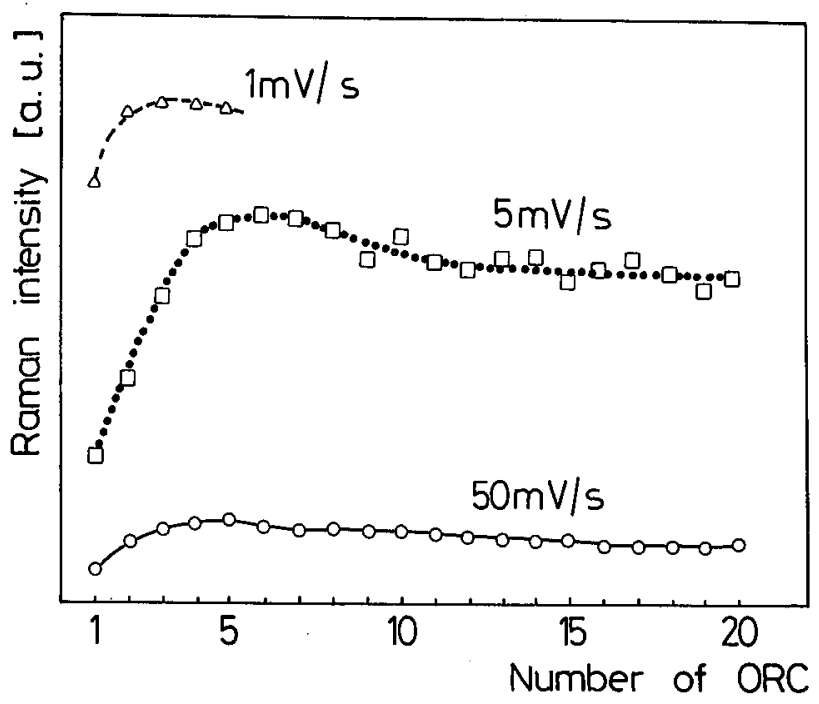

Fig. 6. The dependence of the SERS signal on the number of OR cycles for different sweep rates of the potential on Ag electrode. SERS intensity was measured at the potential equal to $-0.6 \mathrm{~V}_{\mathrm{SCE}}$.

concentration of adatoms and pyridine molecules adsorbed via water. For this reason the intensity of SERS bands is maximal. The physical situation at the $\mathrm{Ag}$ electrode/electrolyte interface in the above range of potential is schematically presented in Fig. 5(a). Decreasing the potentials toward more negative ones, the $\mathrm{Cl}^{-}$ anions and molecules are desorbed, which allows the $\mathrm{Ag}^{0}$ adatoms to diffuse and to be incorporated into the metal (Fig. 5(b)). As a consequence of desorption and absence of adatoms the SERS signal decreases. Applying less negative potentials the pyridine molecules are readsorbed, but the number of $\mathrm{Ag}^{0}$ adatoms becomes small (Fig. 5(c)) and therefore the SERS signal recorded during the increasing potential is much lower than that for the opposite potential sweep. To obtain again the strong SERS signal one has to perform the next oxidation and reduction cycle during which the adatoms will appear again. Volt-Ramanograms presented here proves that the atomic scale roughness, i.e. existence of adatoms at the surface, is very important in SERS phenomenon. From these volt-Ramanograms follows that (for the electrode potential when the pyridine concentration at the surface is the largest), the SERS signal recorded during the decreasing potential (large number of adatoms) is at least one order of magnitude larger than that recorded during the increasing potential (small number of adatoms).

Figure 6 presents the dependence of SERS signal on the number of ORCs and on the sweep rate of potential during ORCs. From this figure it follows that: 1) only a few cycles of ORC are necessary to obtain the maximal SERS signal; 2) the magnitude of the potential sweep rate during ORC is of essential importance. For the slowest sweep we obtain the strongest SERS signal. We suggest that this 


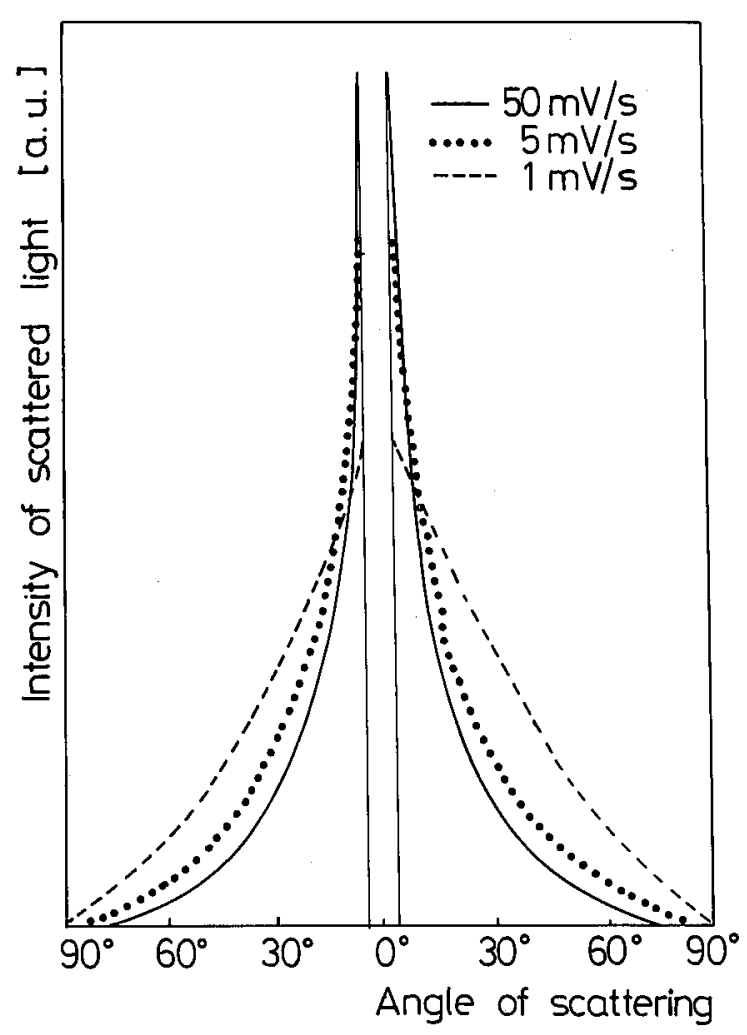

Fig. 7. Angle distribution of the elastically scattered laser light intensity $(\lambda=$ $514.5 \mathrm{~nm}$ ) on silver electrodes with different surface roughness caused by different potential sweep rates.

is caused by the largest microscopic roughness. We assume that during the slowest sweep the most prolongated (large $a / b$ ) bumps are produced on the surface, because in such conditions the largest number of Ag layers are oxidized and next reduced. Accordingly to the EM model we should then obtain the strongest signal. We performed also qualitative investigations of the micro-roughness of electrodes. Figure 7 presents the angle distribution of the intensity of light elastically scattered on the investigated electrodes. From comparison of Figs. 6 and 7 it follows that the least rough surface (small $a / b$ ) gives the weakest SERS signal, whereas the most rough surface (large $a / b$ ) gives the strongest SERS signal. Dependencies presented in Fig. 6 seem to confirm the EM model satisfactorily. It is possible, that SERS signal variations presented in Fig. 6 are caused by the change of both the microand atomic scale roughness. Namely, when the ORC is performed slowly a large number of $\mathrm{Ag}$ layers is oxidized and consequently during the reduction process the prolongated micro-objects as well as the large number of adatoms appears.

In Fig. 6 one can find that after performing a few ORCs we obtain for a 
given sweep rate a maximal value of SERS signal. The next ORCs do not generate an increase of the signal, in contrary, we observe even a small decrease of it. We ascribe this decrease to heating effects $[13,14]$ caused by laser beam irradiation of the electrode during ORCs. Thus, the adatoms could faster diffuse and become incorporated into the lattice.

\section{Conclusion}

We present the results of investigations of the dependence of SERS signal on the silver electrode potential as well as on the sweep rate and on the number of ORCs used to roughen the electrode surface. The presented dependencies prove that the micro- and atomic scale roughness determine the magnitude of the intensity of SERS light. Results presented in Fig. 6, i.e. dependence of SERS signal on the number and sweep rate of ORCs, prove that the micro-roughness has an essential influence on the value of SERS light intensity. This confirms the validity of EM model. On the other hand results presented in Fig. 4, i.e. the SERS signal dependence on the value of the potential and sweep direction after the termination of reduction process (when atomic scale roughness alters only), prove that the CT mechanism produces an additional enhancement of intensity of the Raman scattered light. From our experimental results presented in Fig. 4 follows that the intensity of SERS produced by both CT and EM mechanisms is at least one order of magnitude larger than that produced by the EM mechanism only.

\section{Acknowledgements}

The authors wish to thank doc.dr M. Razwadowski for the help in organizing this work, to dr hab. B. Grzegorzewski for the roughness measurements as well as R. Wegner M.Sc. and J. Konieczka for construction of the electronic circuitry.

\section{References}

[1] M. Fleischman, P.J. Hendra, A.J. McQuillan, Chem. Phys. Lett. 26, 163 (1974).

[2] R.K. Chang, T.E. Furtak, (eds.) Surface Enhanced Raman Scattering, Plenum Press, New York 1982.

[3] R.K. Chang, B.L. Laube, CRC Crit. Rev. Solid State Mat. Sci. 37, 1 (1984).

[4] A. Otto, in Light Scattering in Solids IV, Topics in Applied Physics, eds. M. Cardona, G. Gunntherodt, Vol. 54, Springer-Verlag, Heidelberg 1984, p. 289.

[5] M. Moskovits, Rev. Mod. Phys. 57, 783 (1985).

[6] R.K. Chang, Ber. Bunsenges. Phys. Chem. 91, 296 (1987).

[7] J. Skonieczny, Postepy Fizyki 35, 181 (1984) (in Polish).

[8] P.W. Barber, R.K. Chang, H. Massoudi, Phys. Rev. B 27, 7251 (1983).

[9] P.B. Johnson, R.W. Christy, Phys. Rev. B 6, 4370 (1972). 
[10] A. Otto, J. Billmann, J. Eickmans, U. Erturk, C. Pettenkofer, Surf. Sci. 138, 319 (1984).

[11] T.E. Furtak, D. Roy, Surf. Sci. 158, 126 (1985).

[12] M. Fleischman, I.R. Hill, in Surf. Enhanced Raman Scattering, eds. R.K. Chang, T.E. Furtak, Plenum Press, New York 1982, p. 275.

[13] S.H. Macomber, T.E. Furtak, Solid State Commun. 45, 267 (1983).

[14] J. Qi, G. Wu, Spectrochim. Acta A 45, 711 (1989). 